\title{
Non-contrast computed tomography in the diagnosis of cerebral venous sinus thrombosis
}

\author{
Jernej Avsenik ${ }^{1}$, Janja Pretnar Oblak², Katarina Surlan Popovic ${ }^{1}$ \\ ${ }^{1}$ Clinical Institute of Radiology, University Medical Centre, Ljubljana, Slovenia \\ ${ }^{2}$ Department of Neurology, University Medical Centre, Ljubljana, Slovenia
}

Radiol Oncol 2016; 50(3): 263-268

Received 9 February 2015

Accepted 6 March 2016

Correspondence to: Jernej Avsenik, M.D., Clinical Institute of Radiology, University Medical Centre, Zaloška cesta 7, SI-1000 Ljubljana, Slovenia. Phone: +386 1522 8530; E-mail: jernej.avsenik@gmail.com

Disclosure: No potential conflicts of interest were disclosed.

Background. The aim of the study was to investigate the sensitivity and specificity of non-contrast computed tomography (NCCT) in the diagnosis of cerebral venous sinus thrombosis (CVST).

Methods. Screening our neurological department database, we identified 53 patients who were admitted to neurological emergency department with clinical signs of CVST. Two independent observers assessed the NCCT SCans for the presence of CVST. CT venography and/or MR venography were used as a reference standard. Interobserver agreement between the two readers was assessed using Kappa statistic. Attenuation inside the cerebral venous sinuses was measured and compared between the patient and the control group.

Results. CVST was confirmed in 13 patients. Sensitivity and specificity of NCCT for overall presence of CVST were $100 \%$ and $83 \%$, respectively, with Kappa value of 0.72 (a good agreement between observers). The attenuation values between CVST patients and control group were significantly different (73.4 $\pm 14.12 \mathrm{HU}$ vs. $58.1 \pm 7.58 \mathrm{HU} ; \mathrm{p}=0.000)$. The ROC analysis showed an area under the curve (AUC) of $0.916(95 \% \mathrm{Cl}, 0.827-1.00)$ and an optimal cutoff value of $64 \mathrm{HU}$, leading to a sensitivity of $85 \%$ and specificity of $87 \%$.

Conclusions. NCCT as a first-line investigation has a high value for diagnosis of CVST in the emergency setting. The additional measurement of the sinus attenuation may improve the diagnostic value of the examination.

Key words: cerebral venous sinus thrombosis; computed tomography; stroke

\section{Introduction}

Due to the diversity of underlying factors and the absence of a uniform treatment approach, diagnosis and management of patients with cerebral venous sinus thrombosis (CVST) remain a challenging task. ${ }^{1}$ CVST represents $0.5 \%$ to $1 \%$ of all strokes and affects approximately 5 patients per million every year, but has a higher frequency among younger patients. ${ }^{1-3}$ Typical acquired risk factors include recent surgery, trauma, pregnancy, postpartum state, antiphospholipid syndrome, cancer and use of oral contraceptives. Cases of inherited thrombophilia include Antithrombin III, Protein C and Protein S deficiency, factor V Leiden positivity, prothrombin gene mutation and hyperhomocisteinemia. ${ }^{1-4}$ Infection of parameningeal spaces (ears, paranasal sinuses, oral cavity, face and neck) is common cause of CVST in pediatric population ${ }^{2,5,6}$, but rare in adults. ${ }^{6}$

The symptoms of CVST are not specific. The most common complaint is headache which occurs in up to $90 \%$ of patients. ${ }^{7}$ Additionally, abnormal vision, any of the symptoms of stroke and seizures have been described. ${ }^{7}$ In the past D-dimer levels appeared to be of value as an initial screening test. A study in 2004 evaluated the sensitivity of D-dimer to be $97.1 \%$ and specificity $99.1 \%{ }^{8}$ However, later studies showed that up to $10 \%$ of patients with CVST have a normal D-dimer. ${ }^{9}$

As it is fast, affordable and widely available, non-contrast computed tomography (NCCT) is the most frequently performed imaging study for evaluation of patients with new headache, focal neuro- 


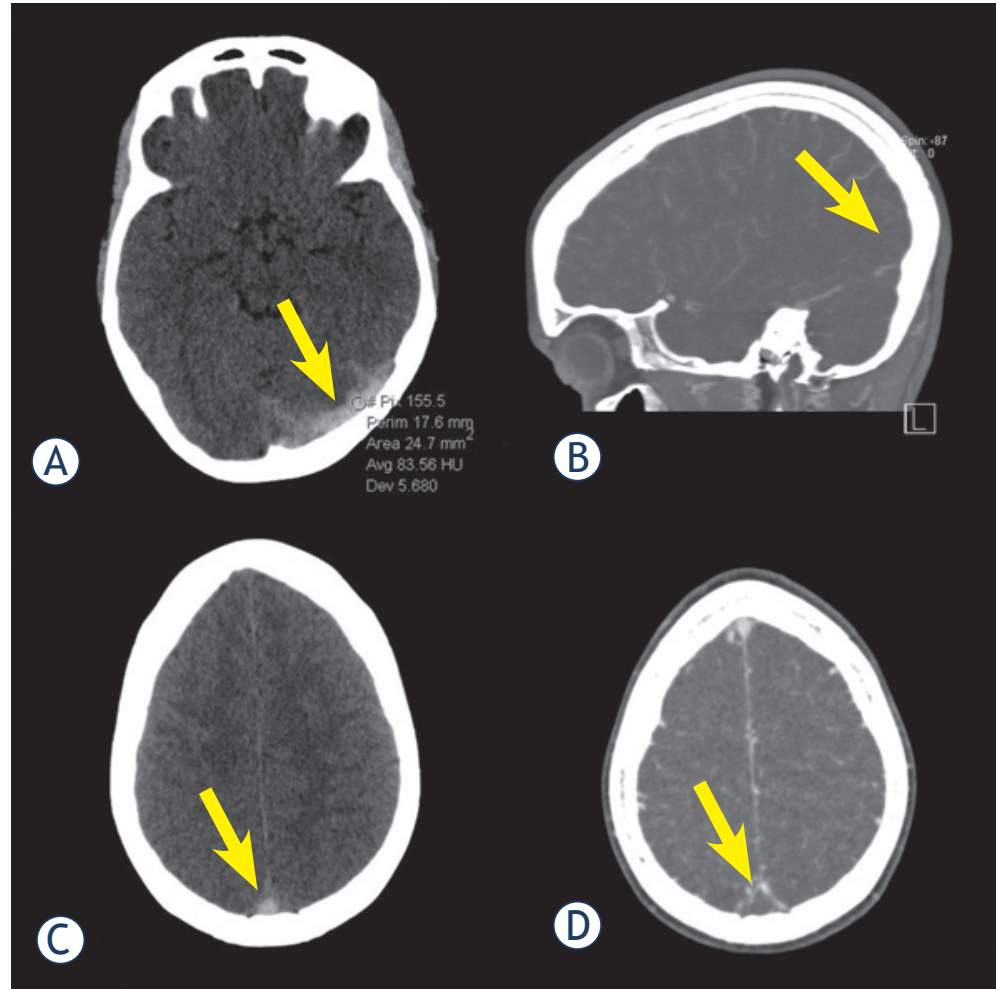

FIGURE 1. 19-year-old female with thrombosis (arrow) of the left transverse sinus (LTS) (A) and superior sagittal sinus (C), confirmed by CT venography in sagittal (B) and axial (D) reconstruction. Average attenuation inside the LTS was $83.6 \mathrm{HU}$.

logical abnormalities, seizure, or change in mental status. A typical imaging finding in patients with CVST is direct visualization of a hyperattenuating thrombus in the occluded sinus (dense sinus sign). ${ }^{2,3}$ Occasionally, NCCT may only show indirect signs of thrombosis, including diffuse brain edema and parenchymal hemorrhage. ${ }^{10}$ Sensitivity of NCCT in the diagnosis of CVST was previously considered rather poor. ${ }^{2,10-13}$ However, using modern multidetector row CT scanners, recent studies report higher sensitivity and specificity values. ${ }^{14}$ In addition, Buyck et al. suggest measurement of the venous sinus attenuation to increase the diagnostic yield of the examination. ${ }^{15}$ Therefore, the goal of the present study was to evaluate the diagnostic accuracy of NCCT in the diagnosis of CVST in the emergency setting.

\section{Patients and methods}

The study was performed in accordance with the Declaration of Helsinki and was approved by the Institutional Review Board Committee.

\section{Subjects}

Screening our neurological department database, we retrospectively identified 53 patients (37 women, 16 men; mean age, 42 years; age range, $17-82$ years) who were admitted to neurological emergency department from July 2008 to May 2013 with clinical signs of CVST. The following inclusion criteria were defined: NCCT had to be performed on admission and at least one of the reference imaging modalities, i.e. CT venography and/or MR venography had to be performed in 24 hours from admission.

\section{CT image acquisition}

All CT images were acquired on Somatom Sensation 40 Open system (Siemens, Erlangen, Germany). The following scanning parameters were used: $120 \mathrm{kV}, 220 \mathrm{mAs}$, section thickness of 3 $\mathrm{mm}$ below the tentorium and $120 \mathrm{kV}, 260 \mathrm{mAs}$ and section thickness of $4.8 \mathrm{~mm}$ above the tentorium.

\section{Image interpretation}

NCCTs were assessed for the presence of direct and indirect signs of CVST by two experienced neuroradiologists, using a standard picture archiving and communication system (PACS) workstation. Observers were blinded to the clinical data and patient identification. Readings were randomized, and standardized evaluation forms were used to ensure the systematic evaluation of the following structures: superior sagittal sinus (SSS), straight sinus (SS), inferior sagittal sinus (ISS), right and left transverse sinuses (RTS, LTS), and right and left sigmoid sinuses, (RSS, LSS). Additionally, right and left internal cerebral vein (RICV, LICV), vein of Galen (VG), right and left basal vein of Rosenthal (BVR), right and left thalamostriate vein (TSV) and cortical veins were assessed. Attenuation inside the thrombosed venous sinus was measured. If no venous structure was classified as thrombosed, mean attenuation of up to three venous sinuses that could be reliably differentiated from surrounding brain parenchyma was documented. Presence of parenchymal hemorrhage or edema was also noted. Finally, observers had to decide regarding the overall presence or absence of CVST and rate their diagnostic confidence on a scale from 1 (absolutely certain) to 5 (uncertain).

After having evaluated all NCCTs, readers reviewed all available imaging data, including follow-ups of any respective patient to obtain a ref- 
erence standard. In this manner, they determined the overall presence of CVST, the involvement of individual venous structures and the presence of parenchymal hemorrhage or edema.

\section{Statistical analysis}

Statistical analysis was performed using IBM SPSS Statistics for Macintosh, Version 20.0. (IBM Corp., Armonk, NY, USA) software package. Based on collected data, we calculated the specificity and sensitivity of NCCT for diagnosis of CVST. Agreement between the two observers was assessed using Kappa statistic. ${ }^{16}$

Patients in whom the diagnosis of CVST was excluded were used as a control group for the second part of the study. Average venous sinus attenuation value of individual subject from the CVST patient group and the control group was used for statistical evaluation. Comparison between the two groups was made by unpaired $\mathrm{t}$-test. Receiver operating characteristic curves (ROC) were used to define optimal cutoff value for which sensitivity and specificity was calculated. A p value $<0.05$ was considered significant.

\section{Results}

CVST was confirmed in 13 patients ( 9 women, 4 men; mean age, 36.5 years; age range, $19-82$ years). Patients presented with headache (12 patients, 93\%), papiloedema (7 of 9 evaluated patients, $78 \%$ ), objective neurological deficit (8 patients, 62\%), vomiting (8 patients, $62 \%$ ), somnolence or stupor (6 patients, $46 \%$ ), confusion ( 5 patients, $38 \%$ ), disturbance of vision (3 patients, $23 \%$ ) and epileptic seizures (2 patients, $15 \%$ ). Risk factors of patients with CVST included oral contraception (6 of 9 female patients), pregnancy, puerperium or recent abortion (4 of 9 female patients). Laboratory values were retrospectively available for 12 of 13 patients. Thrombophilia was found in 6 of 12 evaluated patients (50\%). Average D-dimer value was 2644 $\mathrm{mmol} / \mathrm{L}$, but it was normal in 2 of 12 patients $(17 \%)$. CRP levels were elevated in 9 of 12 patients (75\%).

Transverse sinus was the most commonly thrombosed structure, followed by sigmoid sinus (Table 1). Typical NCCT findings of CVST are depicted in Figure 1. All 13 patients with CVST were accurately diagnosed by both observers. Therefore, the calculated sensitivity was $100 \%$ in both readings, with specificity of 80 and $87.5 \%$, respectively. Kappa value regarding the presence
TABLE 1. Location of thrombus and parenchymal changes in 13 patients with cerebral venous sinus thrombosis (CVST)

\begin{tabular}{lll}
\hline Patient No. & Location of thrombus & Parenchymal changes \\
\hline 1 & RTS, RSS & None \\
2 & LTS & Hemorrhage, edema \\
3 & SSS, RTS, RSS & None \\
4 & RTS & None \\
5 & SSS, RTS, RSS & None \\
6 & SSS, LTS, LSS, right ICV, left ICV, VG & None \\
7 & RTS, RSS & None \\
8 & RTS, RSS & None \\
9 & LTS, LSS & None \\
10 & SSS, RTS, RSS, cortical veins & None \\
11 & SSS, LTS, LSS & Hemorrhage, edema \\
12 & RTS & Hemorrhage, edema \\
13 & SSS, Cortical veins & Hemorrhage, edema \\
\hline
\end{tabular}

ICV = internal cerebral vein; LSS = left sigmoid sinus; LTS = left transverse sinus; $R S S=$ right sigmoid sinus; RTS = right transverse sinus; SSS = superior sagittal sinUs; VG = vein of Galen

or absence of thrombosis was 0.72 , which is considered to represent a good agreement between observers. Sensitivity and specificity of NCCT for overall presence of thrombosis were $100 \%$ and $83 \%$. Average diagnostic confidence level regarding the presence or absence of CVST was $2.1 \pm 1.2$ (very certain). A case with false positive finding is depicted in Figure 2.

Attenuation values were available for all 13 CVST patients and 23 controls and significant difference was found between the two groups (Figure 3). The ROC analysis of the attenuation showed an area under the curve (AUC) of 0.916 (95\% CI 0.827, 1.00) and an optimal cutoff value of $64 \mathrm{HU}$, leading to a sensitivity of $85 \%$ and specificity of $87 \%$.

\section{Discussion}

CVST is a distinct cerebrovascular disorder that, unlike arterial stroke, most often affects young adults and children and is associated with significant morbidity and mortality, especially when the diagnosis is not made in time. ${ }^{17}$ In order to initiate appropriate therapy as soon as possible, early diagnosis is essential.

The clinical presentation of CVST is highly variable. In our series, headache was the most frequently reported symptom. Similar to recent study by Linn et al. ${ }^{14}$, there was no single symptom or sign, present in all patients with CVST. 


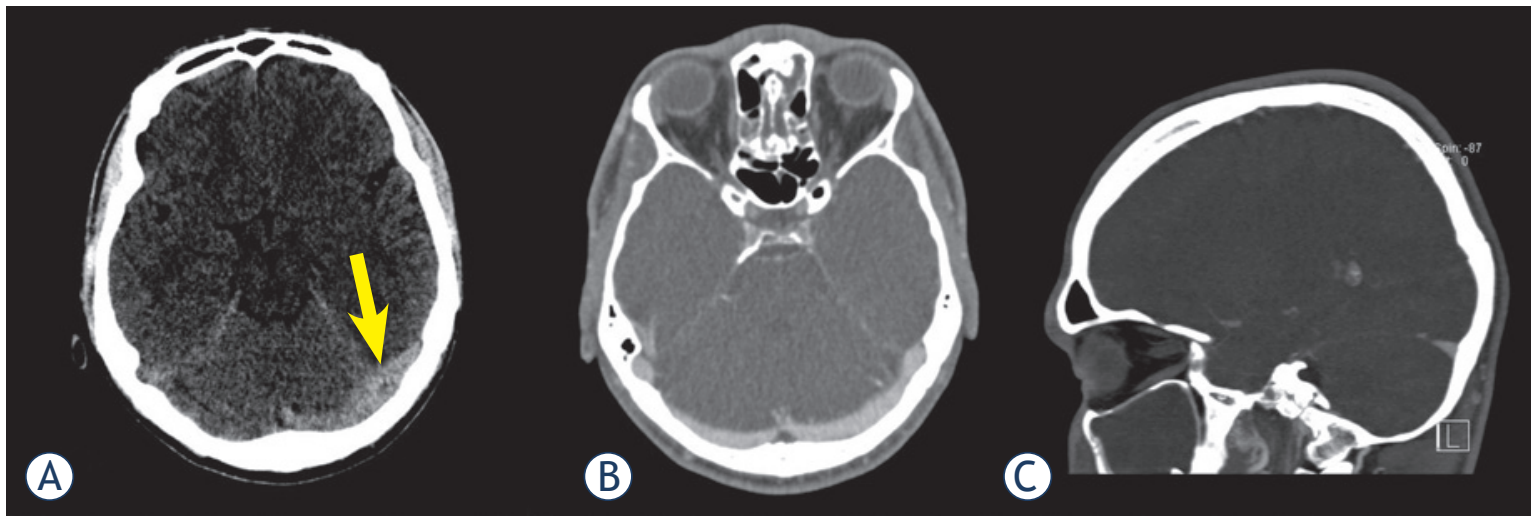

FIGURE 2. Left transverse sinus (LTS) of a 26-year-old male appeared hyperattenuated on non-contrast computed tomography (arrow) (A) and was interpreted as thrombosed by both readers. However, CT venography in axial (B) and sagittal (C) reconstruction showed patency of the LTS. Average attenuation inside the LTS was $60 \mathrm{HU}$.

Normal D-dimer levels have previously been considered to have a high negative predictive value in patients with suspected CVST.7 Recently, normal D-dimer levels were reported in patients with isolated thrombosis of deep cerebral venous system and were explained by the relatively small thrombus volume. ${ }^{14}$ However, we found normal D-dimer levels in 2 patients with CVST, suggesting that D-dimer is of limited value in excluding the diagnosis.

The American Heart Association (AHA)/ American Stroke Association (ASA) 2011 Scientific Statement on diagnosis and management of cerebral venous thrombosis recommends imaging of the cerebral venous system in patients with suspected cerebral venous thrombosis. ${ }^{1}$ Recently, MRI in combination with MR venography has largely replaced digital subtraction angiography as a gold standard for imaging of $\mathrm{CVST}^{17}$ and is currently considered the most sensitive examination technique. ${ }^{18,19}$ In situations when MRI is not readily available, CT venography has been shown to be equivalent to MRI in establishing the diagnosis. ${ }^{20}$ Nevertheless, in most institutions NCCT remains the first-line imaging method in the emergency evaluation of patients with unspecific neurological symptoms ${ }^{14}$, because of its cost-effectiveness and availability. ${ }^{15}$

Using a blinded reader approach, we examined the value of NCCT in diagnosis of CVST, based on presence or absence of classic hyperattenuating signs. These are observed on NCCT scans when an acute thrombus has formed in a blood vessel. The increase in attenuation is caused by clot retraction, eliminating water and thereby raising the concentrations of red blood cells and hemoglobin. This mechanism results in increased attenuation of the thrombus to 60 - 90 HU. ${ }^{15}$ In CVST, hyperattenuating sign (dense sinus sign) can serve as a unique finding indicating an acute stage, at a time when treatment is most likely to be effective and to have a significant effect on clinical outcome. ${ }^{21}$ In the past, sensitivity and specificity of NCCT was considered rather $\operatorname{low}^{11-13}$, and according to the literature, direct signs of CVST were present in only one third of patients. ${ }^{11}$ A study from 1987 reported a sensitivity of approximately $25 \% .{ }^{22}$ However, using modern multidetector row CT scanner, recently reported sensitivity of the direct signs for CVST was $64.6 \%$, which was higher than reported in older studies ${ }^{13,22}$, but still insufficient in excluding the diagnosis. ${ }^{14}$ Authors of this work emphasized the value of NCCT in diagnosis of deep venous thrombosis, reporting the $100 \%$ sensitivity and $99.4 \%$ specificity in this subgroup of patients. In our series however, there was only one patient who, in addition to venous sinus thrombosis, also had deep venous system thrombosis (Table 1).

Our study revealed higher sensitivity $(100 \%)$ of NCCT for overall presence of thrombosis than previously reported $(25 \%-64 \%) .{ }^{13,14,22}$ Specificity, on the other hand, was lower than expected (83.8\%). The observers, although blinded to clinical and imaging data, knew the purpose of the study, which may partially explain these results. Also, if the venous structure was recognized as thrombosed, it was considered a positive finding even in cases, where diagnostic confidence was low (4 or 5 on our scale). Interestingly enough, if we interpreted these cases as negative, the sensitivity and specificity would be $88.5 \%$ and $95 \%$, which is closer to previously published data.

Apparent increase in attenuation of venous sinuses can be misleading and is not always visually 


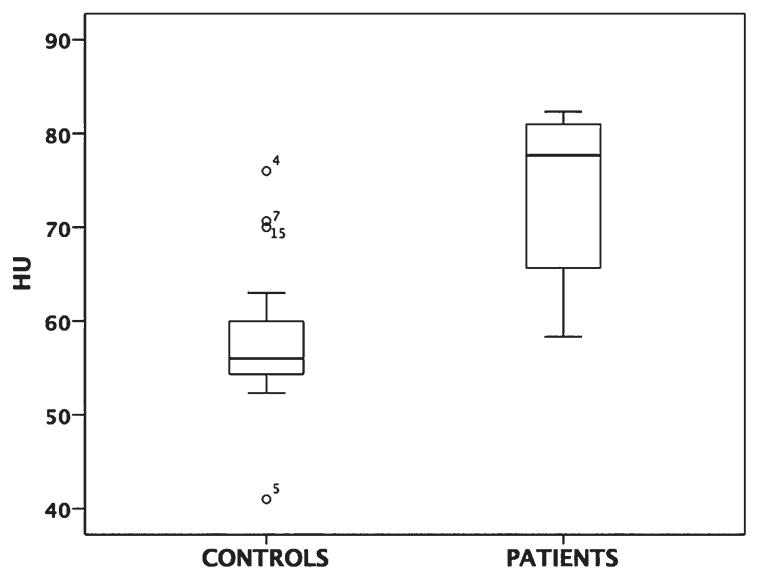

FIGURE 3. The attenuation values between cerebral venous sinus thrombosis (CVST) patients and control group were significantly different $(73.4 \pm 14.12 \mathrm{HU}$ vs. $58.1 \pm 7.58 \mathrm{HU} ; \mathrm{p}=$ 0.000). HU, Hounsfield units.

recognized, therefore recently additional measurement of the sinus attenuation was proposed to increase the sensitivity of the examination in the diagnosis of CVST. ${ }^{15}$ In our series, mean attenuation of 73.4 HU was found in thrombosed venous sinuses. This degree of increased attenuation was previously found in clotted sinuses of patients with $\mathrm{CVST}^{15,23}$, as well as in acute thrombosis elsewhere in the body. ${ }^{23}$ Significant difference in the average sinus attenuation was found between the CVST patient group and the control group. However, an overlap between the two groups and the presence of outliers in the control group (Figure 3) may limit the reliability of this method in some patients. Our results are similar to the findings of Buyck et al., who suggested a threshold of $62 \mathrm{HU}$ to differentiate the patients with CVST from those without. ${ }^{15}$ Based on our data, the optimal threshold was 64 HU. The drawback of this method is the possibility of false negative finding in patients with anemia who have low attenuation of blood due to low hemoglobin. ${ }^{15}$ Similarly, the most common cause of false positive readings is the high attenuation of blood in patients with high hemoglobin or hematocrit level. ${ }^{24}$ These limitations may partially be avoided by calculating the Hounsfield unit-tohematocrit ratio. However, the improvement in accuracy with this method has been shown to be minor. ${ }^{15}$ Additionally, such calculation may often be impractical in emergency setting and was therefore not included in our study.

We acknowledge the following limitations of our work. Relatively small size of the studied population was a drawback. However, CVST is a relatively uncommon disease and the size of our group is comparable to previously published studies. Furthermore, the impact of relatively low number of patients was minimized using the blind and multiple observer approach. DSA was not performed and so the consensus reading of CT venography or MR venography was used as a reference standard. Nonetheless, the diagnostic value of these methods for the diagnosis of CVST has been shown to be very high. ${ }^{55,26}$

In conclusion, prompt therapy of CVST has a profound impact on clinical outcome, therefore early diagnosis is important. Our study has shown high sensitivity of NCCT for diagnosis of CVST and thus confirmed the role of NCCT as a investigation of choice in the emergency setting. The additional measurement of the sinus attenuation may improve the diagnostic value of the examination and help decide on the need for confirmatory study.

\section{References}

1. Saposnik G, Barinagarrementeria F, Brown RD, Jr., Bushnell CD, Cucchiara B, Cushman $\mathrm{M}$, et al. Diagnosis and management of cerebral venous thrombosis: a statement for healthcare professionals from the American Heart Association/American Stroke Association. Stroke 2011; 42: 1158-92.

2. Bousser MG, Ferro JM. Cerebral venous thrombosis: an update. Lancet Neurol 2007; 6:162-70.

3. Ferro JM, Canhao P, Stam J, Bousser MG, Barinagarrementeria F. Prognosis of cerebral vein and dural sinus thrombosis: results of the International Study on Cerebral Vein and Dural Sinus Thrombosis (ISCVT). Stroke 2004; 35: 664-70.

4. de Freitas GR, Bogousslavsky J. Risk factors of cerebral vein and sinus thrombosis. Front Neurol Neurosci 2008; 23: 23-54.

5. Boncoraglio G, Carriero MR, Chiapparini L, Ciceri E, Ciusani E, Erbetta A et al. Hyperhomocysteinemia and other thrombophilic risk factors in 26 patients with cerebral venous thrombosis. Eur J Neurol 2004; 11: 405-9.

6. Damak M, Crassard I, Wolff V, Bousser MG. Isolated lateral sinus thrombosis: a series of 62 patients. Stroke 2009; 40: 476-81.

7. Stam J. Thrombosis of the cerebral veins and sinuses. N Engl J Med 2005; 352: $1791-8$

8. Kosinski CM, Mull M, Schwarz M, Koch B, Biniek R, Schlafer J, et al. Do normal D-dimer levels reliably exclude cerebral sinus thrombosis? Stroke 2004; 35: 2820-5.

9. Crassard I, Soria C, Tzourio C, Woimant F, Drouet L, Ducros A, et al. A negative D-dimer assay does not rule out cerebral venous thrombosis: a series of seventy-three patients. Stroke 2005; 36: 1716-9.

10. Leach JL, Fortuna RB, Jones BV, Gaskill-Shipley MF. Imaging of cerebral venous thrombosis: current techniques, spectrum of findings, and diagnostic pitfalls. Radiographics 2006; 26 (Suppl 1): S19-41; discussion S2-3.

11. Piazza G. Cerebral venous thrombosis. Circulation 2012 125: 1704-9.

12. Renowden S. Cerebral venous sinus thrombosis. Eur Radiol 2004;14: 21526.

13. Teasdale E. Cerebral venous thrombosis: making the most of imaging. $J R$ Soc Med 2000; 93: 234-7.

14. Linn J, Pfefferkorn T, Ivanicova K, Muller-Schunk S, Hartz S, Wiesmann M, et al. Noncontrast CT in deep cerebral venous thrombosis and sinus thrombosis: comparison of its diagnostic value for both entities. Am J Neuroradiol 2009; 30: 728-35. 
15. Buyck PJ, De Keyzer F, Vanneste D, Wilms G, Thijs V, Demaerel P. CT density measurement and $\mathrm{H}: \mathrm{H}$ ratio are useful in diagnosing acute cerebral venous sinus thrombosis. Am J Neuroradiol 2013; 34: 1568-72.

16. Viera AJ, Garrett JM. Understanding interobserver agreement: the kappa statistic. Fam Med 2005; 37: 360-3.

17. Gaikwad AB, Mudalgi BA, Patankar KB, Patil JK, Ghongade DV. Diagnostic role of 64-slice multidetector row $\mathrm{CT}$ scan and $\mathrm{CT}$ venogram in cases of cerebral venous thrombosis. Emerg Radiol 2008; 15: 325-33.

18. Lafitte F, Boukobza M, Guichard JP, Hoeffel C, Reizine D, Ille O, et al. MRI and MRA for diagnosis and follow-up of cerebral venous thrombosis (CVT). Clin Radiol 1997; 52: 672-9.

19. Dormont D, Anxionnat R, Evrard S, Louaille C, Chiras J, Marsault C. MRI in cerebral venous thrombosis. J Neuroradiol 1994; 21: 81-99.

20. Ozsvath RR, Casey SO, Lustrin ES, Alberico RA, Hassankhani A, Patel M Cerebral venography: comparison of CT and MR projection venography. Am J Roentgenol 1997; 169: 1699-707.

21. Goldstein M, Quen L, Jacks L, Jhaveri K. Acute abdominal venous thromboses--the hyperdense CT sign. J Comput Assist Tomogr 36: 8-13.

22. Virapongse C, Cazenave C, Quisling R, Sarwar M, Hunter S. The empty delta sign: frequency and significance in 76 cases of dural sinus thrombosis. Radiology 1987; 162: 779-85.

23. Black DF, Rad AE, Gray LA, Campeau NG, Kallmes DF. Cerebral venous sinus density on noncontrast CT correlates with hematocrit. Am J Neuroradiol 2011; 32: 1354-7.

24. Healy JF, Nichols C. Polycythemia mimicking venous sinus thrombosis. Am J Neuroradiol 2002; 23: 1402-3.

25. Greiner FG, Takhtani D. Neuroradiology case of the day. Superior sagittal sinus thrombosis and infarcts. Radiographics 1999; 19: 1098-101.

26. Wetzel SG, Kirsch E, Stock KW, Kolbe M, Kaim A, Radue EW. Cerebral veins: comparative study of CT venography with intraarterial digital subtraction angiography. Am J Neuroradiol 1999; 20: 249-55. 